\title{
ФУНКЦИОНАЛЬНОЕ МОДЕЛИРОВАНИЕ ПРОЦЕССОВ СИСТЕМЫ ИНДИВИДУАЛЬНОГО ПОДХОДА К ЛЕЧЕНИЮ ПАЦИЕНТОВ С ПРИМЕНЕНИЕМ ТЕХНОЛОГИИ ВIG DАТА
}

\author{
(c) 2020 Миронова Александра Леонидовна \\ студент \\ Сибирский федеральный университет, Россия, Красноярск \\ (c) 2020 Гаврилюк Екатерина Юрьевна \\ студент \\ Сибирский федеральный университет, Россия, Красноярск \\ (c) 2020 Гуслякова Анастасия Владиславовна \\ студент \\ Сибирский федеральный университет, Россия, Красноярск \\ (C) 2020 Свалова Анна Сергеевна \\ студент \\ Сибирский федеральный университет, Россия, Красноярск \\ (c) 2020 Гончар Вероника Николаевна \\ студент \\ Сибирский федеральный университет, Россия, Красноярск
}

Данная статья посвящена информационным технологиям в медицине, а именно применению технологии Big Data для подбора индивидуального лечения пациента, больного онкологическим заболеванием. Рассмотрены причины применения данной технологии, а также исследования, подтверждающие ее эффективность. Проведен анализ системы IBM Watson for Oncology, базирующейся на исследуемой технологии. Осуществлено моделирование системы организации индивидуального варианта лечения онкологически больного пациента с применением технологии Big Data.

Ключевые слова: информационные технологии, Big Data, медицина, лечение, анализ данных, моделирование, аналитика.

В условиях глобальной цифровизации ни одна сфера деятельности человека не остается без внимания к информационным технологиям, реализуемых путем использования множества взаимосвязанных научных и технических областей знания. Этот механизм, ставший доступным благодаря научно-техническому развитию нашего общества, повышает качество жизни человека, значительно упрощая многие технологические процессы.

Концепция Big Data на сегодняшний день является актуальным инструментом информационных технологий по работе с огромными массивами данных во многих проблемных областях, таких как клиентский сервис, финансовые услуги, телекоммуникации, горнодобывающая и нефтяная промышленность и другие. Неоценим вклад данной технологии в медицине, так как в рамках этого направления компьютерные технологии могут использоваться не столько как инструмент для ускорения расчетов, а как помощник, расширяющий человеческие возможности в выборе информации и принятии решений.

Активное внедрение технологии Big Data в медицине связано с новыми тенденциями во взаимоотношениях врача и пациента - в формате технологий персонализированной медицины, ориентированной на конкретного пациента, для которого важны прогнозирование, профилактика заболеваний и индивидуально подобранное лечение.

Сфера здравоохранения не успевает «идти в ногу» с ошеломляющей скоростью появления новой информации, включающей в себя многочисленные данные о лабораторных исследованиях, медицинских испытаниях и различных физиологических параметрах человека. В связи 
с этим концепция Big Data позволяет перейти на принципиально новую ступень в современной медицине - персонализированную и предупреждающую. Анализ накопленных историй болезни и карт диагностики позволит ввести в практику врачей информационные системы поддержки принятия врачебных решений. Алгоритмы машинного обучения, которые умеют находить статистические корреляции в огромных и даже всемирных массивах медицинских данных, откроют возможность оперативно составлять прогнозы и рекомендации для отдельного пациента. Предполагается, что это должно стать вспомогательным инструментом современного лечащего врача и обеспечить высокое качество и эффективность лечения.

Использование Big Data в сфере здравоохранения продиктовано необходимостью решать глобальные проблемы человечества. Население планеты с каждым годом увеличивается на 1,33\%, поэтому прогнозирование эпидемий, борьба с существующими болезнями и усовершенствование методов лечения в целом - злободневные задачи мирового сообщества [1].

В решении насущных проблем можно серьезно продвинуться, взяв за основной инструмент - анализ данных. Уже сегодня технологии сбора и анализа информации позволяют выявить как заболевания отдельных пациентов, так и дать прогноз по поводу состояния здоровья целой социальной группы.

Проведенное исследование Университета Кейптауна, в котором команда исследователей совершила открытие: проанализировав шесть наиболее распространенных видов онкологических заболеваний, подтвердило, что каждый из этих видов рака характеризуется отчетливо выраженной комбинацией генов. Информация об открытии была опубликована 20 мая 2016. Анализ накопленных результатов о состоянии здоровья тысяч пациентов с генетической точки зрения показал, что у рака груди, кишечника, легких, яичников и мозга есть отчетливые генетические маркеры. По словам руководителя исследования, команда не смогла бы совершить открытие, если бы у нее не было доступа к массивам Big Data [2].

Приведенные ранее исследования послужили толчком для разработки уникальной системы IBM Watson. Одним из многочисленных примеров использования системы IBM Watson является помощь докторам в выявлении роли генетического фактора при диагностировании и лечении онкологических и редких патологий. Система анализирует геномные данные с электронной медицинской картой пациента, фильтрует информацию о таких же заболеваниях и, обработав ее, передает данные специалисту [3].

Можно выделить следующие ключевые функции Watson for Oncology:

- Извлечение ключевых атрибутов из медицинской карты пациента (обработка всего содержимого медицинских карт пациентов на естественном языке в случае интеграции с EMR Electronic Medical Record);

- Рекомендации по лечению с доказанной эффективностью (базируются на информации с экспертным обучением Memorial Sloan Kettering Cancer Center для предоставления вариантов лечения с разной степенью достоверности на основе доказательной базы MSK);

- База знаний в области онкологии (дополняет рекомендуемые варианты лечения релевантными доказательными данными из обширной базы знаний, включающей в себя более 300 медицинских журналов, 250 учебников и 15 миллионов страниц текста, и обрабатываемой на естественном языке с помощью расширенных алгоритмов машинного обучения);

- Анализ данных, ориентированный на пациента, который осуществляет поиск дополнительных доказательных данных в статьях, соответствующих характеристикам пациента и рассматриваемым вариантам его лечения.

Перед созданием модели определим контекст моделирования, включающий в себя субъект моделирования, цель моделирования и точку зрения на модель.

Субъектом является сама система индивидуального подхода к лечению пациента, больного онкологией.

Цель моделирования - создать наглядный образец системы выполнения медицинского процесса «Подбор индивидуального лечения онкологически больного пациента».

После определения контекста моделирования можно приступать к построению контекстной диаграммы в нотации IDEF0, иногда называемой «черным ящиком». Данный тип диаграммы позволяет показать, что подается на вход работы и что является результатом работы, без детализации ее составляющих. Данная диаграмма (рисунок 1) содержит только одну работу, которая будет представлять изучаемый процесс 
в целом. В данной системе - это «Подбор индивидуального лечения для пациента с онкологическим заболеванием».

Инициирующим событием является обращение пациента с жалобами за медицинской помощью, именно оно подается на вход (рисунок 1). Результатом процесса является составленное врачом-онкологом индивидуальное лечение для пациента с учетом рекомендаций по лечению с доказанной эффективностью от Watson for Oncology.

Рассматриваемый медицинский процесс регулируется следующими нормативноправовыми актами: Приказом Министерства здравоохранения РФ от 15 ноября 2012 г. N 915н «Об утверждении Порядка оказания медицинской помощи населению по профилю «онкология» (с изменениями и дополнениями) и Федеральным законом от 27.07.2006 N 152-Ф3 «О персональных данных». Также элементами управления на диаграмме являются должностная инструкция врача-онколога, содержащая функциональные обязанности врача и порядок их выполнения, медицинская практика лечащего врача и его коллег в современном онкологическом центре, в котором функционируют информационные системы и базы данных с постоянно пополняющейся медицинской информацией данного центра и медицинская наука, включающая в себя медицинскую литературу, постоянно обновляющиеся рекомендации, передовые методики, исследования, медицинские журналы, учебники и статьи.

Основные механизмы, выполняющие работу, это - врач-онколог, оборудование для диагностики рака, необходимое на диагностическом и эндоскопическом этапах обследования больного, а также Watson for Oncology, программное обеспечение, оценивающее информацию из медицинской карты пациента, обращающееся к медицинским знаниям и выдающее потенциальные варианты лечения с разными уровнями достоверности вместе с доказательной базой. Врач-онколог может выбрать оптимальную стратегию лечения, исходя из своего профессионального опыта.

После создания контекстной диаграммы (A-0), которая представляет собой описание контекста моделируемой системы, проводится функциональная декомпозиция: система разбивается на подсистемы и каждая подсистема описывается в том же синтаксисе, что и система в целом.

Рассмотрим полученную диаграмму декомпозиции (рисунок 2).

Первым этапом после обращения пациента с жалобами является контактная работа врача с пациентом (A1). Данный блок регулируется должностной инструкцией врача-онколога, Приказом Министерства здравоохранения РФ и медицинской практикой, которая, в свою очередь, регулирует все блоки данного уровня декомпозиции. Механизмами выполнения данной работы являются врач-онколог и оборудование

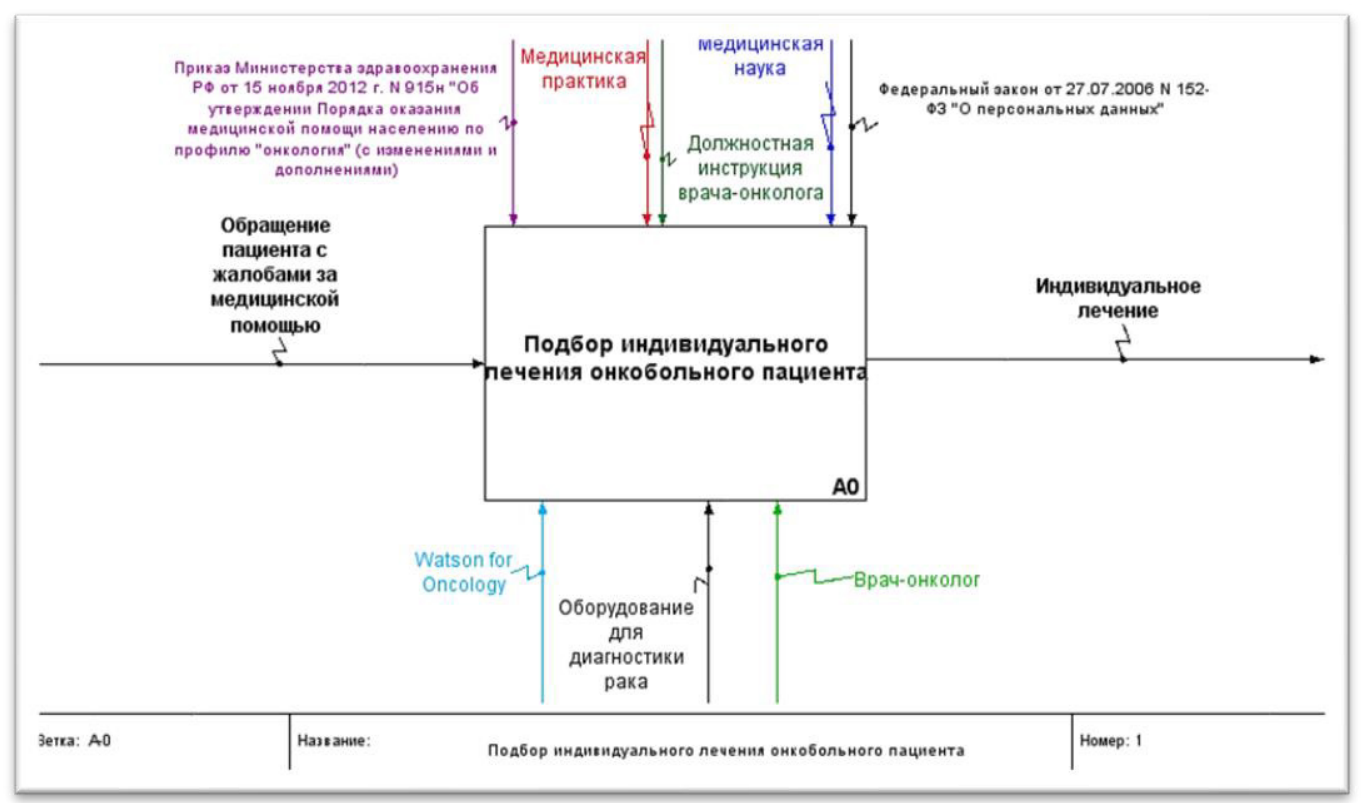

Рисунок 1. Контекстная диаграмма А-0 


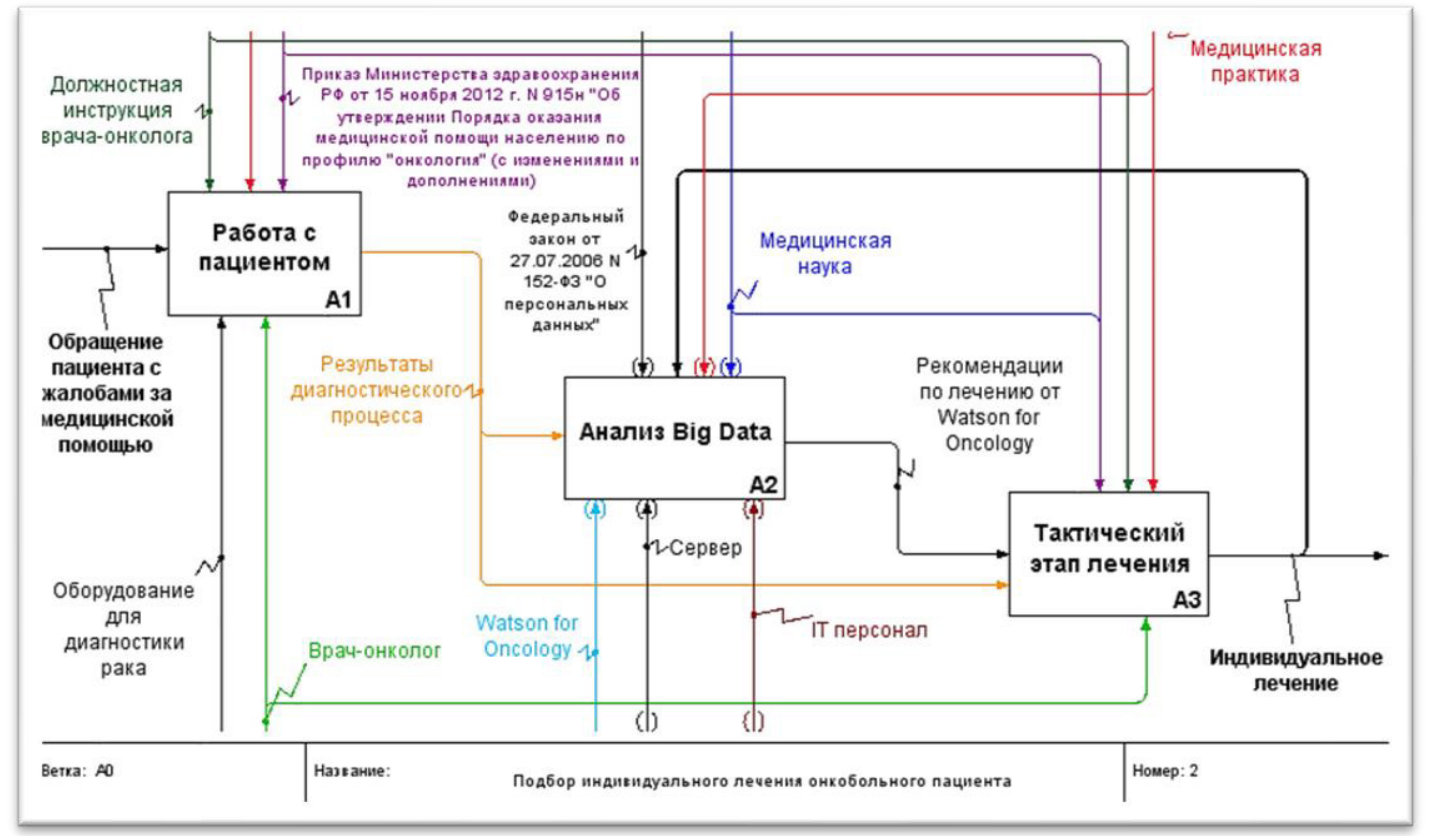

Рисунок 2. Диаграмма декомпозиции уровня А0

для лабораторной диагностики рака. Результат выполнения данной работы в декомпозиции результаты диагностического процесса, которые являются входящей информацией для следующего блока - «Анализ Big Data».

Блок «Анализ Big Data» (A2) представляет собой автоматизированную аналитическую составляющую медицинского процесса, так как в рамках цифровизации компьютерные технологии могут использоваться не столько как инструмент для обработки и хранения больших объемов медицинской информации, а как помощник, расширяющий возможности врача в выборе информации и принятии решений. В дальнейшем данный блок должен быть продекомпозирован в нотации DFD, так как является исключительно информационным процессом.

Как видно на рисунке 2, результатом данного процесса являются рекомендации по лечению от Watson for Oncology, управлением - Федеральный закон о персональных данных, упомянутые выше медицинская наука и практика, выступающие в роли обучающей медицинской информации для IBM Watson, а также индивидуальное лечение, полученное в результате медицинского процесса.

Важно отметить тот факт, что результат функционирования всей системы - индивидуальное лечение, то, зачем обращался пациент к врачу, является ценной информацией, полученной в рамках концепции Big Data. Данный факт является особенностью моделируемой системы, так как полученный результат может функционировать как управление модели, которым при обращении уже другого пациента с похожим анамнезом может обучаться и в дальнейшем руководствоваться Watson for Oncology, накапливая тем самым свою базу знаний в области онкологии.

Аналитика в рамках Big Data невозможна без следующих механизмов: IT-персонала, выполняющего функцию управления информационными системами, Watson for Oncology - ПО, предоставляющего рекомендации по лечению, и сервера, на котором находятся все данные, необходимые для полноценного функционирования баз данных и платформы IBM Watson.

Тактический этап (A3) в разработке индивидуального лечения для онкологически больного пациента является заключающим. Он регулируется должностной инструкцией врача-онколога, медицинской практикой и медицинской наукой. Данный процесс непосредственно выполняет врач-онколог, после анализа полученной информации из предыдущих этапов, устанавливая причинно-следственные связи и уточняя окончательный диагноз, он разрабатывает план дальнейшего лечения.

Таким образом, была создана модель современной системы, способной подбирать индивидуальное лечение с учетом рекомендаций системы IBM Watson for Oncology для пациента, 
больного онкологическим заболеванием.

Была смоделирована система, в которой осуществляется весь цикл лечения от обращения пациента с жалобами к врачу до создания плана индивидуального лечения с учетом всех информационных подсистем, используемых в данном медицинском процессе.

Данный цикл гарантирует более точный и эффективный подбор лечения, так как позволяет создать модель персонализированной медицины, в которой благодаря технологии Big Data учитываются индивидуальные физиологические показатели каждого пациента, а не обезличенные статистические данные, накопленные годами и потерявшие свою актуальность для современной цифровой медицины.

В ходе выполнения курсовой работы было выявлено, что использование Big Data может изменить способ управления, анализа и использования данных в медицинской сфере. Но при этом, внедрение технологии Big Data сегодня затруднено в связи с некоторыми факторами, например:

- У медицинских центров нет большой заинтересованности делиться информацией о пациентах друг с другом, что снижает потенциал для накопления и обработки Big Data. Ho, когда они начнут получать деньги на основе положительных результатов лечения пациентов, а не за сам факт оказания помощи то появится финансовый стимул для обмена данными, которые могут быть использованы для улучшения жизни пациентов при сокращении расходов на страховые компании.

- Отсутствие единого стандарта ведения истории болезни также препятствует реализации данной технологии. Информационным компаниям необходимо создать единый протокол обмена медицинскими данными, которого пока нет. Чем больше будет доступной и стандартизованной медицинской информации из самых разных стран мира, тем точнее будет аналитическое описание болезней и прогнозы.

- Обилие лишней информации: примерно 78\% медицинских данных не структурированы, фильтровать и анализировать такие объе- мы информации сложно. Сам сбор информации сегодня ничего не стоит: хранение данных обходится дешевле, чем уничтожение. Но обилие несущественной информации может привести аналитические системы к ложным выводам. Например, о ложной зависимости между возникновением заболевания и внешними факторами [4].

Несмотря на трудности в реализации технологии Big Data в медицинской сфере, шаг навстречу цифровизации медицины в нашей стране сделан.

Так, в 2016 году в РФ был сформирован ГОСТ Р 52636-2006, который определяет параметры электронной медицинской карты. А в 20202025 годах Минздрав в сотрудничестве с Ростехом готовят масштабное внедрение Единой государственной информационной системы в здравоохранении (ЕГИСЗ), предназначенной, в частности, для «развития систем электронного медицинского документооборота и внедрения единых стандартов информационного обмена в этой сфере» [5].

В связи с вышесказанным можно обозначить следующие перспективы во внедрении рассмотренной системы по созданию индивидуального подхода к лечению и развитии технологии Big Data в рамках здравоохранения в целом.

Медицина достаточно консервативная область и всё новое внедряется с некоторой задержкой, поэтому полезно медицинским структурам иногда осматриваться и следить, как другие отрасли справляются с Big Data, извлекая из них пользу. Они могут вдохновить на появление прогрессивных идей, их быстрое внедрение и адаптацию. Здравоохранению необходимо догнать другие отрасли, которые уже перешли от стандартных методов, основанных на регрессии, к более ориентированным на будущее, таким как интеллектуальная аналитика. Специалистам здравоохранения необходимо тщательно собирать медицинские данные и искать лучшие стратегии для их использования, примером которых может стать система по созданию индивидуального подхода к лечению онкологически больного пациента.

\section{Библиографический список}

1. Big Data в здравоохранении: как и для чего медицина использует Большие Данные? [Электронный ресурс]: DataSides | Whatever you want to know about Data Science.- Режим доступа: http://ru.datasides.com/big-datamedicine/\#_nbsp 
2. IBM Watson for Oncology [Электронный ресурс]: IBM Российская Федерация.- Режим доступа: https://www. $\mathrm{ibm}$.com/ru-ru/marketplace/clinical-decision-support-oncology

3. Когнитивная система IBM Watson Health - прорыв в сфере здравоохранения [Электронный ресурс]: Mentamore - информационно-познавательный портал.- Режим доступа: https://mentamore.com/ covremennye-texnologii/kognitivnaya-sistema-ibm-watson-health-proryv-v-sfere-zdravooxraneniya.html

4. Применение Big Data в медицине [Электронный ресурс]: Блог Medical Note о здоровье и цифровой медицине.- Режим доступа: https://blog.mednote.life/articles/technology/primenenie-big-data-v-medicine

5. Big data на страже здоровья: как и зачем медицинские организации собирают и хранят данные [Электронный ресурс]: Хайтек - Медиа про высокие технологии в России и лучшие мировые практики.-Режим доступа: https://hightech.fm/2018/09/21/bigdata-med 